\title{
Enhancement of Oxygen Contrast in a STEM HAADF Image of Perovskite Oxide SrTiO3 Using Maximum Entropy Method
}

\author{
L. Xie ${ }^{1,2}$ and X. Q. Pan ${ }^{1,3}$
}

1. College of Modern Engineering and Applied Science, Nanjing University, Nanjing China 210093.

2. Department of Materials Science and Engineering, University of Michigan, Ann Arbor MI, USA 48105.

3. Department of Physics, University of California Irvine, Irvine CA, USA 92697.

Functional oxides of perovskite structure are of great research interest both for their fundamental and novel physical properties, e.g. multiferroic, metal-insulator transition, two-dimensional electron gas and polar metal, as well as versatile industrial applications, for example, piezoelectric motors, actuators, ultrasound sensors, ferroelectric tunnel junction, etc.. Oxygen atoms, which constitute the cornerstone of perovskites, play an important role in the peculiar physical properties of these materials due to their strong orbital hybridization and interaction with cations. Therefore, a thorough understanding of oxygen atoms is essential for the understanding of structure-property relationships and the design of novel functional oxides and related devices.

Owing to its small atomic number, it is once difficult to directly observe oxygen in transmission electron microscopy (TEM). Only recently, with the advance of aberration-corrected TEM, direct characterization of oxygen atoms at atomic scale becomes available in TEM, for instance, negative Cs imaging [1], annular bright-field imaging [2] and electron energy-loss spectroscopy [3]. However, there are only a few reports that discuss the observation of oxygen in high angle annular dark field (HAADF) images due to the relatively weak scattering factor of oxygen. In this paper, we show that the contrast of oxygen atoms in oxides of perovskite structure can be enhanced by deconvolution of the HAADF image using Maximum Entropy Method (MEM) [4].

Figure 1(a) displays a typical atomic-resolution HAADF image of $\mathrm{SrTiO}_{3}$, viewed along the [001] direction, in which $\mathrm{Sr}$ and $\mathrm{Ti}$ atoms are of bright contrast but $\mathrm{O}$ atoms cannot be observed by eye. The original image was then deconvoluted using the MEM algorithm with software package STEM_CELL [5]. The parameters used for deconvolution correspond to the experimental values of our aberrationcorrected STEM JEOL-3100 R05 equipped with a cold field-emission gun, with an accelerating voltage of $\mathrm{V}=300 \mathrm{kV}$, convergence semi-angle equals to $22 \mathrm{mrad}, \mathrm{C} 3=0.5 \mu \mathrm{m}, \Delta f=-12.2 \AA$ and a source size about $0.7 \AA$ A. As is shown in Figure 1(b) is the deconvoluted result, in which not only the Sr and Ti atoms can be seen clearly, but also the contrast of $\mathrm{O}$ atoms can be readily observed. In order to further understand the origin of the contrast of $\mathrm{O}$ atoms in the deconvoluted image, we compare the line profiles along one of the Ti-O atomic chains and the results are shown in Figure 1(c). It should be noted that the peaks corresponding to oxygen atomic columns actually already exist in the original HAADF image. So that it's not a surprise that the deconvoluted image could exploit such hidden information.

A possible explanation for the occurrence of the oxygen contrast in the original HAADF image is that the local potential of the oxygen atom is rather shallow, and as a result, the Debye-Waller factor of oxygen is relatively larger than those of cations. It's worth pointing out that in addition to $\mathrm{SrTiO}_{3}$, we also observed oxygen contrast in the deconvoluted HAADF images for a series of perovskites, such as $\mathrm{BaTiO}_{3}, \mathrm{BiFeO}_{3}$ and $\mathrm{GdScO}_{3}$. Therefore, it's suggested that Z-contrast imaging combined with MEM 
deconvolution provides a possible route for the atomic-resolution characterization of oxygen in a wide variety of functional oxides. Quantitative image simulation is ongoing to gain understanding of the underlying mechanism for the origin of oxygen contrast in HAADF images.

\section{References:}

[1] CL Jia, M Lentzen and K Urban, Science 299 (2003), p. 870.

[2] E Okunishi et al, Microscopy and Microanalysis 15 (2009), p. 164.

[3] SJ Pennycook and PD Nellist, "Scanning Transmission Electron Microscopy", (Springer, New York).

[4] PD Nellist and SJ Pennycook, Journal of Microscopy 190 (1997), p. 159.

[5] V Grillo, Microscopy and Microanalysis 17 (2011), p. 1292.
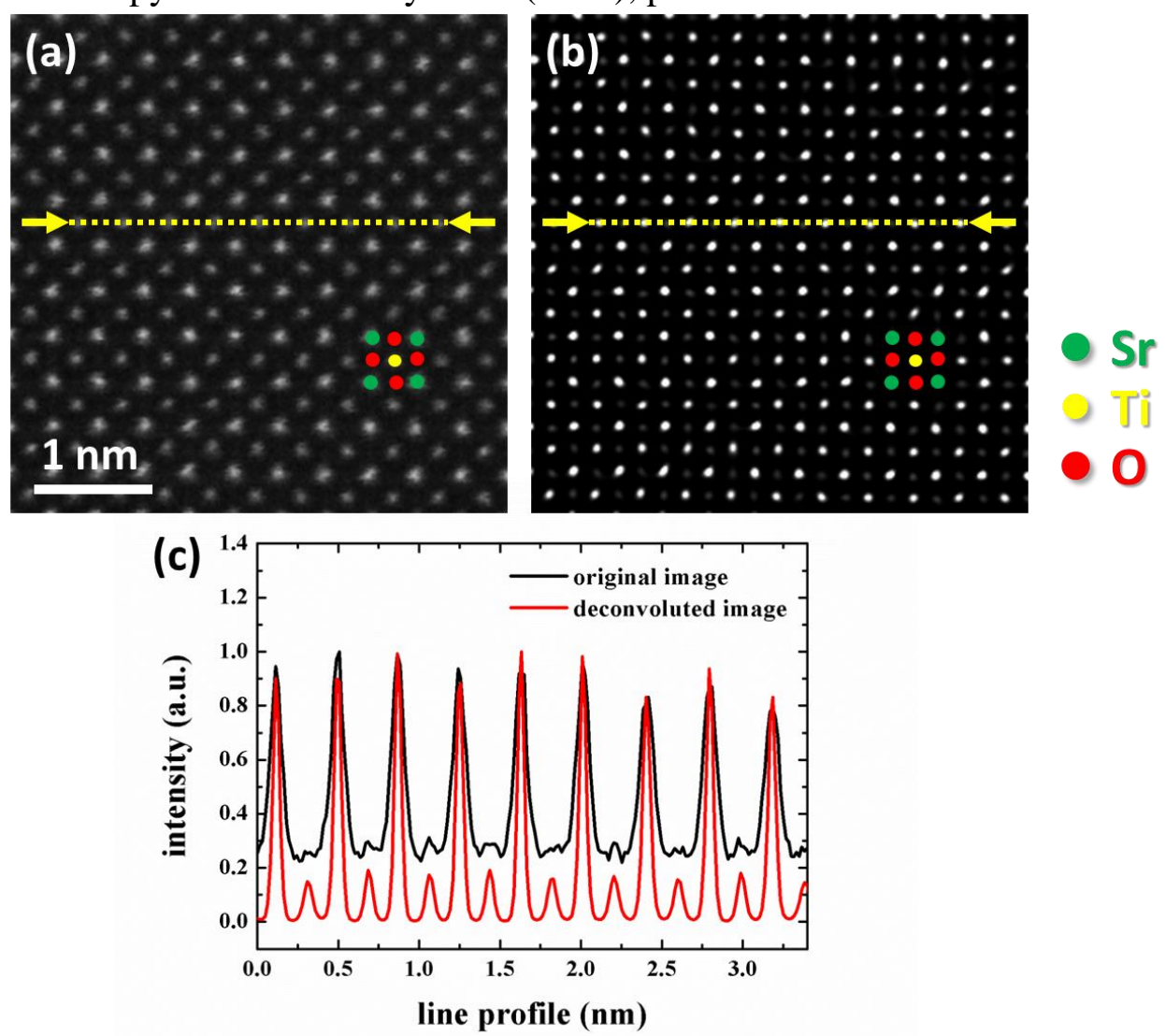

Figure 1. (a) the original and (b) the deconvoluted $\mathrm{Z}$-contrast image of $\mathrm{SrTiO}_{3}$ viewed along [001] zone axis. (c) line profiles of the yellow lines in (a) and (b). The faint oxygen contrast can barely be seen in the line profile of original image but can be seen clearly after the deconvolution. 